\title{
DIAGNÓSTICOS DE ENFERMAGEM PARA PACIENTES COM TUBERCULOSE
}

\section{NURSING DIAGNOSES FOR TUBERCULOSIS PATIENTS}

\author{
Maiana Evillyn da Silva Santos ${ }^{1}$ Luana Nunes dos Santos $^{2}$ Danielly Cardoso de Mesquita ${ }^{3}$ Joseilze $^{2}$ \\ Santos de Andrade ${ }^{4}$ Lígia Mara Dulce de Lemos $^{5}$ Flávia Janólio Costacurta Pinto da Silva ${ }^{6}$
}

\section{RESUMO}

Objetivo: sintetizar a produção científica sobre diagnósticos de enfermagem para pacientes com tuberculose. Método: trata-se de um estudo bibliográfico, descritivo, tipo revisão integrativa da literatura, sem limite temporal, realizado por meio das bases de dados: LILACS, BDENF, IBECS, PUBMED, Portal Periódicos Capes e na Biblioteca Virtual SciELO. Utilizou-se os descritores: "tuberculosis"; "nursing diagnosis", "nursing process" e "nursing care". Resultados: foram identificadas 875 publicações; após o processo de seleção resultaram três artigos como amostra final. Seguida a análise dos estudos, verificou-se 60 diagnósticos de enfermagem relacionados aos pacientes com tuberculose. Em dois artigos avaliados, a Teoria das Necessidades Humanas Básicas foi o embasamento teórico do processo de enfermagem. Constatou-se que dentre as classificações de linguagens padronizadas de enfermagem, a mais utilizada foi a CIPE, seguida pela NANDA. Houve a predominância de estudos desenvolvidos em nível de assistência terciária e de estudo de caso. Considerações finais: $O$ enfermeiro ao aplicar o seu julgamento clínico tem autonomia de levantar para os pacientes com tuberculose diversos diagnósticos de enfermagem, utilizando-se desse instrumento de trabalho para elaboração de planos de cuidados eficazes para o público-alvo em questão.

Palavras-chave: Tuberculose; Saúde Pública; Processo de Enfermagem; Diagnóstico de Enfermagem; Cuidados de Enfermagem.

\begin{abstract}
Objective: to synthesize the scientific production on nursing diagnoses for patients with tuberculosis. Method: this is a bibliographic, descriptive study, integrative literature review type, without time limit, carried out through the following databases: LILACS, BDENF, IBECS, PUBMED, Capes Periodicals Portal and the SciELO Virtual Library. The following descriptors were used: "tuberculosis"; "nursing diagnosis", "nursing process" and "nursing care". Results: 875 publications were identified; after the selection process, three articles resulted as the final sample. Following the analysis of the studies, 60 nursing diagnoses related to patients with tuberculosis were found. In two articles evaluated, the Basic Human Needs Theory was the theoretical basis of the nursing process. It was found that among the classifications of standardized nursing languages, the most used was the ICNP, followed by NANDA. There was a predominance of studies developed at the level of tertiary care and case study. Final considerations: When applying their clinical judgment, nurses have the autonomy to raise different nursing diagnoses for patients with tuberculosis, using this working tool to develop effective care plans for the target audience in question.
\end{abstract}

Keywords: Tuberculosis; Public Health; Nursing Process; Nursing Diagnosis; Nursing Care

\footnotetext{
${ }^{1}$ https://orcid.org/0000-0002-6485-7375. Universidade Federal de Sergipe (UFS), Aracaju (SE), Brasil

${ }^{2}$ https://orcid.org/0000-0002-7237-9625. Universidade Federal de Sergipe (UFS), Aracaju (SE), Brasil

${ }^{3}$ https://orcid.org/0000-0002-5551-1479. Universidade Federal de Sergipe (UFS), Aracaju (SE), Brasil

${ }^{4}$ https://orcid.org/0000-0003-0488-2840. Universidade Federal de Sergipe (UFS), Aracaju (SE), Brasil

${ }^{5}$ https://orcid.org/0000-0003-0676-8478. Universidade Federal de Sergipe (UFS), Aracaju (SE), Brasil

${ }^{6}$ https://orcid.org/0000-0002-9866-5654. Universidade Federal de Sergipe (UFS), Aracaju (SE), Brasil https://doi.org/10.31011/reaid-2021-v.95-n.35-art.1111 Rev Enferm Atual In Derme v. 95, n. 35, 2021 e-021098
} 


\section{INTRODUÇÃO}

A Tuberculose (TB) ainda persiste como um problema de saúde pública no Brasil e no mundo, pois apresenta altas taxas de morbidade relacionadas, principalmente, ao abandono e a resistência ao tratamento ${ }^{(1)}$. Essa enfermidade relaciona-se com fatores determinantes de saúde como aglomerações populacionais, condições socioeconômicas precárias, desnutrição, alcoolismo, dependência química, doenças ou condições imunossupressoras $^{(2)}$.

A TB é uma das 10 principais causas de morte em todo mundo. Em 2019 vitimou 1,4 milhão de pessoas e outras 10 milhões adquiriram a doença a nível global. Conforme Relatório Global da Tuberculose 2020, a tendência de alta da incidência de TB no Brasil, durante os anos de 2016 a 2019, está preocupando a situação nas Américas ${ }^{(3)}$.

O continente americano apresentou aproximadamente $3,0 \%$ da carga mundial de TB, com 268 mil casos novos estimados em 2015, sendo o Brasil o país com a maior parcela, representando 33,0\% em relação à América, em seguida destacam-se Peru $(14,0 \%)$ e México $(9,0 \%)^{(4)}$. Em 2017, o Brasil ocupou a $20^{\mathrm{a}}$ colocação no ranking dos 30 países de alta carga para TB considerados prioritários pela Organização Mundial da Saúde (OMS) para o controle da doença no mundo $^{(5)}$.

O coeficiente de mortalidade no Brasil
$2018^{(6)}$. Em 2019, foram notificados 96.005 casos no país, com um coeficiente de incidência de aproximadamente 45,6 por 100 mil habitantes brasileiros, sendo São Paulo o Estado mais afetado pela doença, com 21.909 novos casos. Em contrapartida, o Estado do Tocantins foi o que apresentou menor incidência, equivalente a 233 casos no mesmo ano ${ }^{(7-8)}$.

Na perspectiva de promover o controle da doença no âmbito da saúde pública, tornase essencial a assistência de enfermagem por meio da busca ativa e da identificação dos determinantes de saúde relacionados à tuberculose, possibilitando o diagnóstico precoce da doença, assim como por meio de intervenções que estimulem a adesão do paciente ao tratamento completo ${ }^{(9-10)}$.

Para que haja sucesso nas ações de enfermagem relacionadas ao controle da TB no Brasil, é imprescindível que o cuidado prestado à população seja sistematizado e integrado às atividades da saúde pública ${ }^{(11-12)}$. Nesse sentido, o processo de enfermagem (PE) se caracteriza como o principal instrumento facilitador do trabalho da enfermagem, o qual é operacionalizado por meio de etapas interligadas, interdependentes e inter-relacionas ${ }^{(13)}$. Ressalta-se que o diagnóstico de enfermagem (DE) caracterizase como um fator norteador do cuidado de enfermagem, pois permite que o enfermeiro identifique as necessidades de intervenção

foi de 2,2 óbitos/100mil habitantes em 
para cada paciente, de forma individualizada e holística $^{(11)}$.

É importante que a prática profissional do enfermeiro seja respaldada em fundamentações teóricas, elas transmitem ao enfermeiro um arcabouço teórico para diagnosticar, tratar, reabilitar, prevenir e promover saúde da população assistida ${ }^{(14-15)}$. A utilização dos Sistemas de Linguagens Padronizadas (SLP) de enfermagem durante a prática assistencial é fundamental para a consolidação da mesma enquanto ciência e profissão $^{(16)}$, sendo a Classificação Internacional para a Prática de Enfermagem (CIPE) e a North American Nursing Diagnosis Association (NANDA) as mais empregadas durante a aplicação do PE no Brasil $^{(17)}$.

\section{OBJETIVO}

Sintetizar a produção científica sobre diagnósticos de enfermagem para pacientes com tuberculose.

\section{MÉTODO}

Trata-se de um estudo bibliográfico, descritivo, do tipo revisão integrativa da literatura, composta pelas etapas: identificação do tema e definição da pergunta condutora; pesquisa nas bases bibliográficas após estabelecimento dos critérios de inclusão e exclusão; coleta dos dados; análise e interpretação dos artigos incluídos e por fim apresentação da revisão/síntese de conhecimento $^{(18)}$.

Elaborou-se a seguinte questão norteadora: "Quais os principais diagnósticos de enfermagem para pacientes com tuberculose?". Adotou-se, em seguida, os seguintes critérios de inclusão: artigos originais disponíveis de forma gratuita que apresentassem diagnósticos de enfermagem para pacientes com tuberculose, publicados nos idiomas português, inglês ou espanhol, sem recorte temporal e sem restrição de nacionalidade de publicação. Excluíram-se estudos que não contemplassem a pergunta norteadora, estudos de revisão, cartas ao editor, editoriais e anais de evento científico.

A coleta de dados foi realizada entre os meses de novembro de 2020 a janeiro de 2021, por três autoras do estudo, nas bases de dados: Literatura Latino-Americana e do Caribe em Ciências da Saúde (LILACS), Banco de Dados em Enfermagem (BDENF) e Índice Bibliográfico Espanhol de Ciências da Saúde (IBECS) através da Biblioteca Virtual da Saúde (BVS); National Library of Medicine (PUBMED); Scientific Electronic Library On-line (SciELO) e Portal Periódicos Capes. Utilizou-se para a busca, os seguintes descritores contemplados no Descritores em Ciências da Saúde (DeCS) e no Medical Subject Heading (Mesh) em combinação, por meio dos operadores booleanos AND e OR: "tuberculosis” AND ("nursing diagnosis" OR "nursing process" OR “nursing care”). 
Conforme ilustrado na Figura 1, foram identificados 875 registros na busca inicial, dos quais foram excluídos aqueles em duplicidade e indisponíveis, totalizando 805. Após leitura dos resumos dos demais 70

Figura 1- Fluxograma da seleção dos estudos adaptado do Preferred Reporting Items for Systematic Reviews and Meta-Analyses (PRISMA 2009). Aracaju (SE), Brasil, 2021.

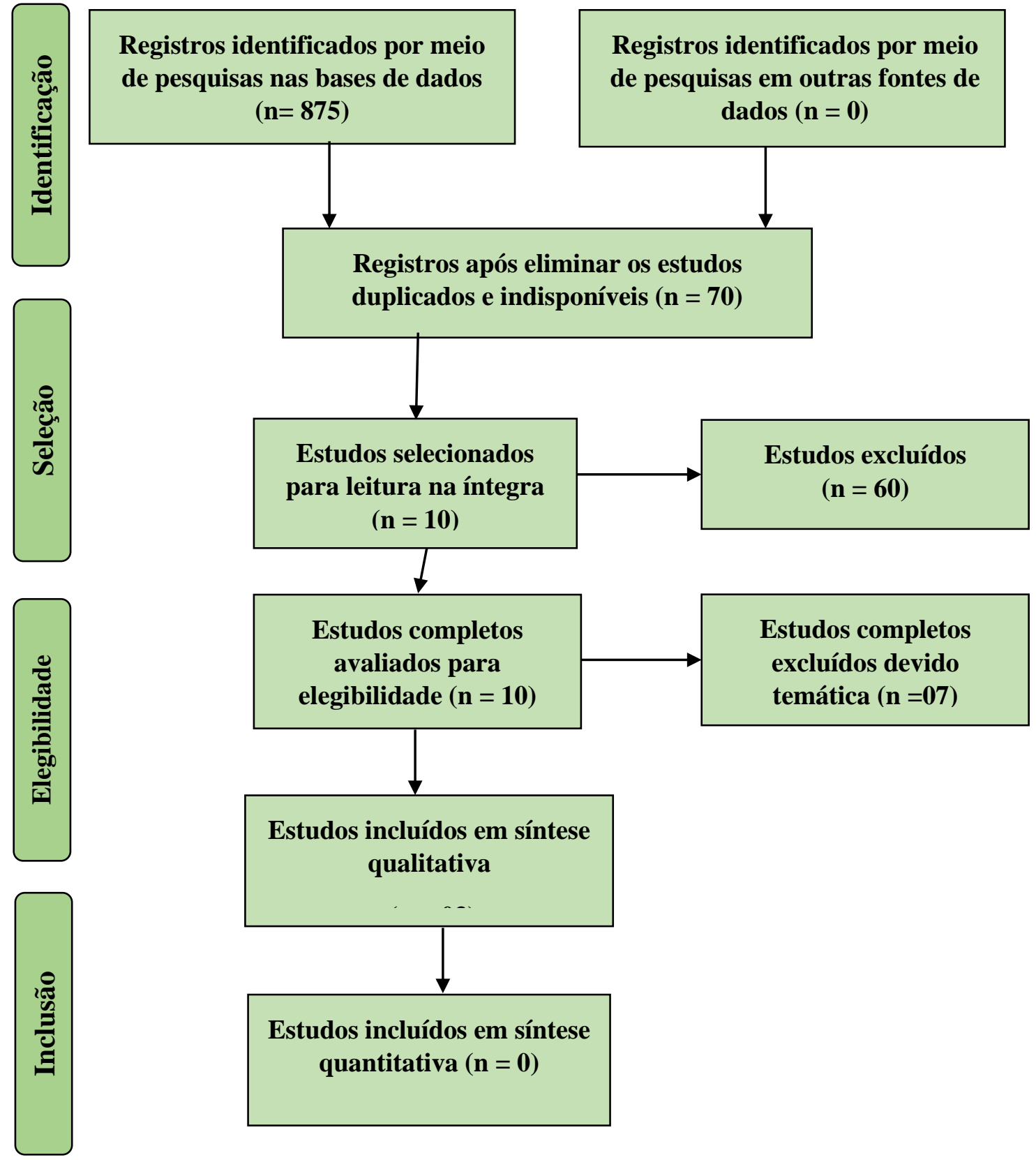

Fonte: Os autores artigos, 60 foram excluídos pois não contemplavam a pergunta norteadora, 10 foram lidos na íntegra e, destes, três foram incluídos na síntese qualitativa. 
Para extração e organização dos dados, foi utilizado instrumento elaborado por Ursi $^{(19)}$ e adaptado pelos autores contendo as seguintes variáveis: título, local, ano e periódico de publicação, tipo de estudo, principais aspectos e resultados relacionados ao tema da pesquisa (linguagens padronizadas, diagnósticos de enfermagem, teoria de enfermagem, nível de atenção à saúde) e nível de evidência científica.

Para classificação do nível de evidência dos estudos selecionados utilizou-se o instrumento do Critical Apprailsal Skills Programme (CASP) adaptado $^{(20)}$, categorizando-os em seis níveis, segundo os critérios estabelecidos: Nível I - metanálise ou revisão sistemática; Nível II - estudo experimental ou randomizado; Nível III estudos não randomizados ou caso-controle; Nível IV - pesquisa descritiva, qualitativa, revisões bibliográficas; Nível V - relatos de caso ou dados de avaliação de programas;
Nível VI - opinião de autores ou relatórios de comitês de especialista.

Os artigos foram avaliados quanto ao rigor metodológico, criticamente, utilizandose o instrumento adaptando do $\mathrm{CASP}^{(21)}$ após análise por meio da leitura reflexiva e criteriosa acerca das principais informações e elementos que compõem a temática nos estudos. Com a análise dos artigos acerca das principais informações e elementos que compõem a temática nos estudos, notou-se que os aspectos discutidos se correlacionam com o seu referencial teórico, resultando a última etapa com a publicação do estudo.

\section{RESULTADOS}

Nesta revisão incluíram-se três artigos científicos originais, todos publicados em português e na região Nordeste do Brasil. As publicações ocorreram entre os anos de 2016 a 2019. A seguir, apresenta-se o panorama geral dos estudos analisados (Quadro 1).

Quadro 1- Distribuição dos artigos conforme ano, autores, periódico, base de dados, tipo do estudo e nível de evidência. Aracaju (SE), Brasil, 2021.

\begin{tabular}{|c|ccc|}
\hline \multicolumn{4}{|c|}{$\begin{array}{c}\text { Artigo (A) } \\
\text { A1 }\end{array}$} \\
A2 - Diagnósticos de enfermagem para pacientes com tuberculose na unidade de \\
infectologia
\end{tabular}

https://doi.org/10.31011/reaid-2021-v.95-n.35-art.1111 Rev Enferm Atual In Derme v. 95, n. 35, 2021 e-021098 


\begin{tabular}{|c|c|c|c|c|}
\hline A1 & 2019 & $\begin{array}{l}\text { Neto, Vinícius Lino de Souza; Da Silva, } \\
\text { Richardson Augusto Rosendo; Fonseca, } \\
\text { Ivana de Araújo; Santos, Sergilene Fonseca } \\
\text { Teixeira; Dos Santos, Anzielyson Felix } \\
\text { Silva; Do Nascimento, Ana Gláucia Lopes }\end{array}$ & $\begin{array}{c}\text { Rev. Cult. } \\
\text { Cuid } \\
+ \\
\text { IBECS- BVS }\end{array}$ & $\begin{array}{c}\text { Estudo de } \\
\text { caso } \\
+ \\
\text { Nível V }\end{array}$ \\
\hline A2 & 2018 & $\begin{array}{c}\text { Guimarães, Tânia Maria Rocha; Amorim, } \\
\text { Camila Torres; Barbosa, Emanuella } \\
\text { Fernanda França; Silva, Fabíola Mirellys } \\
\text { da; Farias, Cynthia Emanuelle Lima; Lopes, } \\
\text { Bianka Santos }\end{array}$ & $\begin{array}{l}\text { Rev. pesqui. } \\
\text { cuid. fundam. } \\
\quad(\text { Online }) \\
\quad+ \\
\text { IBECS- BVS }\end{array}$ & $\begin{array}{c}\text { Estudo de } \\
\text { caso } \\
+ \\
\text { Nível V }\end{array}$ \\
\hline A3 & 2016 & $\begin{array}{c}\text { Renan Rossoni, Eliane de Fátima Almeida } \\
\text { Lima, Carolina Maia Martins Sales, } \\
\text { Cândida Caniçali Primo }\end{array}$ & $\begin{array}{l}\text { Rev enferm } \\
\text { UFPE on line } \\
+ \\
\text { BDENF- } \\
\text { BVS }\end{array}$ & $\begin{array}{c}\text { Estudo } \\
\text { descritivo } \\
+ \\
\text { Nível IV }\end{array}$ \\
\hline
\end{tabular}

Fonte: Os autores.

No que se refere a utilização de teorias de enfermagem, identificou-se, nos artigos A1 e A2, o uso do referencial teórico de Wanda Horta como embasamento para o processo de enfermagem, enquanto que autores do estudo A3 não mencionaram nenhuma teoria de enfermagem.

No tocante à utilização dos Sistemas de Linguagens Padronizadas de enfermagem, foram citadas a NANDA no artigo A2 e a CIPE - em A1 e A3. Quanto ao nível de atenção e locais em que os estudos foram desenvolvidos, verificou-se que A1 e A2 abordaram o nível de atenção terciária em
Hospitais Escolas. $\mathrm{O}$ artigo $\mathrm{A} 3$ não foi desenvolvido em campo assistencial, pois se tratou de um estudo metodológico.

Os três estudos analisados descreveram um total de 60 diagnósticos de enfermagem de acordo com as taxonomias NANDA e CIPE. Para análise destes, foram distribuídos de acordo as necessidades humanas básicas preconizadas pela Teoria das Necessidades Humanas Básicas de Wanda Horta (Quadro 2).

Quadro 2- Distribuição dos diagnósticos conforme a Teoria de Wanda Horta. Aracaju (SE), Brasil, 2021.

\begin{tabular}{|ccc|}
\hline $\begin{array}{c}\text { Necessidades Humanas } \\
\text { Básicas }\end{array}$ & Diagnósticos de Enfermagem \\
\hline $\begin{array}{c}\text { Necessidades } \\
\text { psicobiológicas: }\end{array}$ & NANDA & CIPE \\
Oxigenação & $\begin{array}{l}\text { Padrão respiratório ineficaz; Abuso de tabaco; dispneia } \\
\text { ventilação espontânea prejudicada; }\end{array}$ & $\begin{array}{l}\text { leve; dispneia moderada; } \\
\text { dispneia }\end{array}$ \\
\hline
\end{tabular}




\begin{tabular}{|c|c|c|}
\hline & troca de gases prejudicada; fadiga & 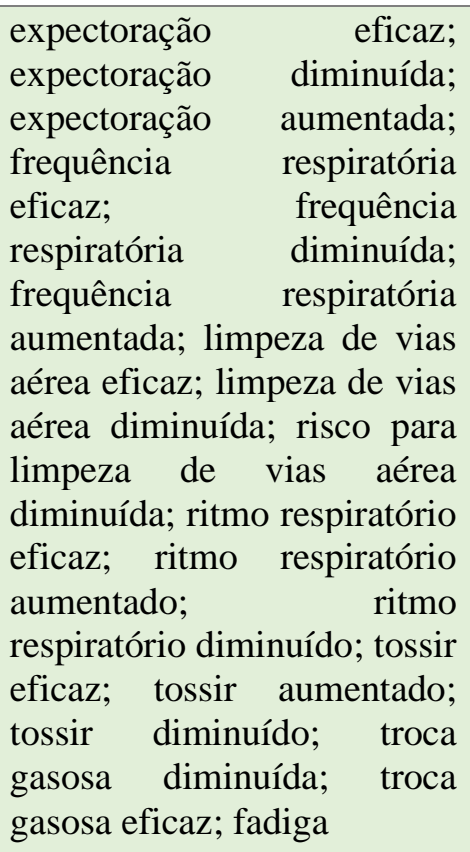 \\
\hline Hidratação & & Suor real \\
\hline Nutrição & $\begin{array}{l}\text { Nutrição desequilibrada: menos do } \\
\text { que as necessidades corporais }\end{array}$ & $\begin{array}{l}\text { nutrição } \\
\text { prejudicada; } \\
\text { diminuído; risco para peso } \\
\text { corporal diminuído }\end{array}$ \\
\hline Sono e repouso & Fadiga & Fadiga; insônia \\
\hline $\begin{array}{l}\text { Exercício e atividades } \\
\text { físicas }\end{array}$ & & $\begin{array}{l}\text { Intolerância à atividade leve; } \\
\text { intolerância à atividade } \\
\text { moderada; intolerância à } \\
\text { atividade severa; risco para } \\
\text { intolerância à atividade; } \\
\text { fadiga leve; fadiga modera; } \\
\text { fadiga severa. }\end{array}$ \\
\hline Abrigo & Manutenção do lar prejudicada & \\
\hline Cuidado corporal & & $\begin{array}{l}\text { Higiene da cavidade oral } \\
\text { prejudicada; padrão de } \\
\text { higiene do couro cabeludo } \\
\text { comprometido }\end{array}$ \\
\hline $\begin{array}{c}\text { Regulação: térmica, } \\
\text { hormonal, neurológica, } \\
\text { hidrossalina, eletrolítica, } \\
\text { imunológica, crescimento } \\
\text { celular, vascular. }\end{array}$ & & $\begin{array}{l}\text { Edema periférico; febre real; } \\
\text { suor real; calafrio real }\end{array}$ \\
\hline $\begin{array}{l}\text { Percepção: olfativa, visual, } \\
\text { auditiva, tátil, gustativa, } \\
\text { dolorosa. }\end{array}$ & Dor crônica & $\begin{array}{ll}\text { Náusea leve; náusea } \\
\text { moderada; náusea severa; } \\
\text { dor visceral leve; dor } \\
\text { visceral moderada; dor } \\
\text { visceral severa; }\end{array}$ \\
\hline Ambiente & Manutenção do lar prejudicada & \\
\hline
\end{tabular}




\begin{tabular}{|clc|}
\hline $\begin{array}{c}\text { Terapêutica } \\
\text { Necessidades } \\
\text { psicossociais: } \\
\text { Gregária }\end{array}$ & Manutenção ineficaz da saúde & Risco para infecção cruzada \\
Espaço & NANDA de solidão & CIPE \\
Aceitação & Manutenção do lar prejudicada & Isolamento social \\
Auto-estima & Risco de suicídio & \\
$\begin{array}{c}\text { Auto-imagem } \\
\text { Necessidades }\end{array}$ & Distúrbios da identidade pessoal & Baixa autoestima \\
$\begin{array}{c}\text { psicoespirituais: } \\
\text { Religiosa ou teológica, ética da identidade pessoal } \\
\text { ou de filosofia de vida. }\end{array}$ & $\begin{array}{l}\text { Tristeza;angústia espiritual; risco } \\
\text { de suicídio. }\end{array}$ & \\
\hline
\end{tabular}

Fonte: Os autores

\section{DISCUSSÃO}

Verificou-se uma reduzida quantidade de artigos publicados que abordaram os diagnósticos de enfermagem para pacientes diagnosticados com tuberculose, o que pode estar associado às lacunas existentes na formação acadêmica dos enfermeiros tanto em relação ao processo de enfermagem quanto ao manejo da $\mathrm{TB}^{(23-24)}$. A quantidade reduzida de publicações acerca desta temática, assim como o fato de as mesmas serem realizadas entre 2016 e 2019 demonstram a fragilidade deste assunto no campo da enfermagem, sugerindo-se assim, a necessidade de mais estudos que contemplem estes temas.

A metodologia estudo de caso foi predominante dentre os estudos (A1 e A2), seguida de um estudo descritivo (A3), resultado semelhante foi mencionado em outra revisão integrativa que caracterizou publicações que abordaram o tema referente à aplicação do processo de enfermagem no Brasil $^{(17)}$. Embora o método estudo de caso corresponda ao nível $\mathrm{V}$ de evidência científica, os resultados procedentes dessa metodologia podem contribuir para a construção de conhecimentos de evidência científica mais relevantes, pois possibilita, por meio de uma abordagem descritiva, que o pesquisador compreenda fenômenos complexos a respeito do objeto investigado ${ }^{(20,}$ 25-26).

O delineamento de pesquisa utilizado em A3, estudo caracterizado como descritivo pelos autores, tratou-se, na verdade, de um estudo metodológico para construção de um protocolo assistencial com diagnósticos e intervenções de enfermagem para pacientes acometidos por TB. Nota-se que o referido tipo de estudo é fortemente utilizado em pesquisas que objetivam a elaboração de instrumentos, protocolos, manuais, escalas e guias, além da validação de aplicativos ou 
semelhantes, pois proporciona aos enfermeiros pesquisadores mecanismos aprimorados para obtenção de dados, análise de resultados confiáveis e testes rigorosos para avaliar intervenções ${ }^{(27-29)}$.

Em relação aos estudos realizados em âmbito assistencial, observou-se que o nível predominante foi o terciário, sendo que as publicações analisadas realizaram suas pesquisas em Hospitais Escolas. Tal resultado pode estar associado à fragilidade da aplicação do PE nos outros níveis de atenção à saúde, principalmente na atenção primária, a qual deve ser o nível assistencial mais resolutivo para esta enfermidade. Vale ressaltar que a TB é considerada como um problema de saúde pública que envolve um tratamento prolongando e determinantes de saúde que possuem manejo mais adequado em nível da atenção básica ${ }^{(1,17,30-31)}$.

Dentre os estudos que aplicaram o PE em campo assistencial, A1 utilizou a CIPE e A2 a NANDA como embasamento para a construção dos diagnósticos de enfermagem, já o artigo A3, optou ao uso da CIPE. Assim como em outros estudos ${ }^{(17,32)}$, o uso da CIPE foi predominante, o que pode estar relacionado ao fato de que essa classificação engloba diagnósticos, resultados e intervenções de enfermagem em um único exemplar, sendo este aspecto um facilitador de seu manuseio ${ }^{(33)}$, diferentemente da NANDA que aborda apenas os diagnósticos. Entretanto, outros autores apresentaram https://doi.org/10.31011/reaid-2021-v.95-n.35-art.1111 Rev Enferm Atual In Derme v. 95, n. 35, 2021 e-021098 resultados discordantes, em que o uso da NANDA foi preeminente, sendo a mesma utilizada mundialmente ${ }^{(34)}$.

Observou-se, nesta revisão, que os dois estudos de casos analisados utilizaram a teoria das Necessidades Humanas Básicas de Wanda de Aguiar Horta para embasarem sua prática clínica. $\mathrm{O}$ artigo A3 não mencionou modelo teórico de embasamento para o processo de enfermagem. Ressalta-se a importância da aplicação desta teoria para assistência de enfermagem a pessoa diagnosticada com $\mathrm{TB}$, pois é por meio dela o enfermeiro é capaz de enxergar o paciente de forma holística, uma vez que esta teoria considera os aspectos psicobiológico, psicossociais e psicoespirituais do ser humano, confrontando assim o modelo biomédico de diagnóstico e tratamento de doenças que ainda persiste ${ }^{(35)}$.

No estudo A3 não foi mencionado modelo teórico de enfermagem para fundamentação do processo de enfermagem, entretanto, foi proposto pelos autores, a construção de um protocolo assistencial com base na terminologia da CIPE. Para a elaboração do documento, os autores realizaram uma revisão de literatura com o objetivo de coletar dados relevantes sobre os aspectos fisiopatológicos da TB e dos cuidados de enfermagem necessários para atender a esse público. Como resultado, apresentaram 39 diagnósticos e 143 
intervenções relacionados à assistência de enfermagem ao paciente com tuberculose.

Considerando os aspectos psicobiológicos da teoria de Horta, a necessidade de oxigenação foi predominante nos pacientes diagnosticados com TB, o que está relacionado com a fisiopatologia da doença, uma vez que a tuberculose pulmonar é preponderante em relação aos demais tipos $^{(1)}$. Além disso, a sintomatologia patognomônica da tuberculose contribui para que haja uma insuficiência no processo de oxigenação do corpo, demando assim, necessidade de um número maior de cuidados de enfermagem para suprir essa necessidade ${ }^{(1,}$ 11-12). Ainda levando-se em consideração a fisiopatologia da TB e os seus sinais e sintomas de febre vespertina e sudorese noturna $^{(1)}$ observa-se que esses achados resultaram na elaboração dos $\mathrm{DE}$ categorizados nas necessidades de hidratação e regulação.

Dentro dos aspectos psicobiológico foram identificados DE referentes à necessidade de nutrição. A relação entre a tuberculose e a desnutrição é um aspecto bem estabelecido e debatido na literatura, sendo a perda de peso um dos sintomas principais do paciente com $\mathrm{TB}^{(1)}$. A subnutrição energéticoprotéica tem alta prevalência em adultos com TB piorando o perfil imunológico e aumentando a morbidade e mortalidade, pois está correlacionada à evolução da doença e insucesso no tratamento ${ }^{(36)}$. Dentro do contexto de declínio da resposta imunológica está associado também o elevado risco de infecção cruzada $^{(36)}$, aplicado a tal risco foi-se trabalhado o DE "risco de infecção" apresentado nas necessidades terapêuticas.

Em uma correspondência publicada na sessão Infectious Diseases no The Lancet em 2020 foi incentivado a inclusão nos futuros estudos epidemiológicos marcadores de nutrição como o índice de massa corporal (IMC), circunferência do braço ou escore Z, como variáveis preditores da doença. Devendo assim, os programas de atenção à tuberculose incluir uma triagem para desnutrição e interpretá-la com cautela ${ }^{(37)}$.

Nos artigos avaliados neste estudo também foram traçados DE relacionados as necessidades de exercício e atividade física e sono e repouso conforme teoria de Horta. Nota-se uma estreita relação entre os DE dessas necessidades, excetuando-se o diagnóstico de insônia, com os DE das necessidades de oxigenação e nutrição, visto que para ter energia para o desenvolvimento de atividades é preciso nutrientes e oxigênio.

Outra categoria de DE evidenciada nesta revisão foi das necessidades de percepção, sendo eles relacionados a dor e náuseas. Tais DE podem estar relacionadas à própria doença, bem como a efeitos adversos do tratamento medicamentoso ${ }^{(1)}$. É responsabilidade do profissional enfermeiro respeitar as percepções dos pacientes e prestar um cuidado humanizado na tentativa de 
minimizar ou até mesmo solucionar os sinais e sintomas desagradáveis ao bem-estar do ser humano ${ }^{(38)}$.

Para as necessidades psicossociais e psicoespirituais foram identificados DE segundo ambas as taxonomias, NANDA e CIPE. Esses diagnósticos corroboram com os achados característicos das pessoas que vivem com tuberculose, nas quais, não se manifestam apenas alterações físicas, mas por se tratar de uma patologia multifacetada, também são passiveis de acometimento psíquico, social e espiritual ${ }^{(39-40)}$.

A doença muitas das vezes gera sofrimento, não só ao ser portador da TB, mas também ao seu nicho familiar. Autores ${ }^{(41)}$ constataram que a doença, assim como o tratamento trazem sofrimento e que o abandono do tratamento da TB está diretamente relacionado a tal condição. Concluíram que essa representação social- "a doença e o tratamento trazem sofrimento"assim denominada por eles, está vinculada a três aspectos: "a tomada de medicamentos é ruim pelos seus efeitos colaterais", “o abandono do tratamento causa/leva à morte" e "o abandono do tratamento dar-se pela falta de apoio social".

Assim, o sofrimento causado pela própria doença e o seu tratamento que está interligado de maneira direta ou indireta aos DE listados nas categorias das necessidades psicossociais e psicoespirituais merece total atenção, sendo um fator causador do https://doi.org/10.31011/reaid-2021-v.95-n.35-art.1111 Rev Enferm Atual In Derme v. 95, n. 35, 2021 e-021098 abandono do tratamento, repercute não só nos aspectos pessoais de quem vive com a doença, mas também para a sociedade no que tange o controle e erradicação da $\mathrm{TB}^{(41-42)}$.

Diversas são as reações emocionais vivenciadas pelos indivíduos infectados pela $\mathrm{TB}$, podendo destacar o medo, o choque, a depressão e o isolamento, notando-se relação direta dessas emoções com os DE trazidos nesta revisão ${ }^{(43)}$. A manutenção ineficaz da saúde foi um dos diagnóstico de enfermagem apresentado, definido como a incapacidade de identificar, controlar e/ou buscar ajuda para manter o bem-estar, está caracterizado e relacionado a essas reações ${ }^{(44)}$.

O significado da doença é influenciado pela história e contexto socioeconômico do qual o portador faz parte, podendo surgir a discrição/exclusão, a morte e a pobreza. O estigma e o preconceito relacionado à tuberculose levam a diversas implicações, desde a ocultação da doença ao abandono do tratamento ${ }^{(41,43)}$. Existe uma forte relação desse contexto socioeconômico com o DE da NANDA "manutenção do lar prejudicado", uma vez que o ambiente sem condições sanitárias, dificuldade de manter um ambiente confortável, além da crise financeira estão correlacionados.

Conforme o Manual de Recomendações para o controle da TB no Brasil $^{(1)}$ o sucesso do controle da doença está associado também ao enfrentamento da discriminação e do preconceito, a fim de 
eliminar barreiras e ampliar o acesso a bens e serviços públicos. Nesse contexto, o profissional enfermeiro deve ser capacitado e apto a desempenhar o seu papel centrado no cuidado integral ao ser humano. O suporte fornecido pelo enfermeiro deve ser adequado as reais necessidades e expectativas do portador de TB.

\section{CONSIDERAÇÕES FINAIS}

Esta revisão pretendeu, ao abordar o processo de enfermagem e analisar especificamente a produção do conhecimento sobre diagnósticos de enfermagem para pacientes com $\mathrm{TB}$, pontuar os $\mathrm{DE}$ que podem ser considerados pelos enfermeiros na assistência ao portador de TB, utilizando-se das taxonomias CIPE e NANDA, além de remeter a importância desse instrumento de trabalho para contribuição e elaboração de planos de cuidado eficazes para o públicoalvo em questão.

Ao distribuir tais DE conforme as necessidades humanas básicas preconizadas na Teoria de Wanda Horta, foi possível notar que a oxigenação e a nutrição se apresentaram como as necessidades psicobiológicas que requerem mais cuidados de enfermagem para com os pacientes com tuberculose. Além disso, os esforços do profissional enfermeiro, devem estar voltados para sanar as necessidades psicoespirituais e psicossocias das pessoas acometidas pela TB, principalmente as relacionadas à autoestima $\mathrm{e}$ à gregária.

Vale ressaltar a importância e aplicabilidade do DE para uma satisfatória condução do paciente com TB ao tratamento. Embora a quantidade de artigos fosse mínima, os diagnósticos de enfermagem apresentados nesses mesmos artigos somam-se 60 e, portanto, são dados relevantes o suficiente para análise geral.

Quanto às limitações do estudo, podese mencionar que os resultados e discussão dos achados foram restritos, fato atribuído ao reduzido número de artigos que abordaram o tema investigado, pela análise de artigos disponibilizados gratuitamente e na íntegra, assim como, a exclusão de teses e dissertações sobre o assunto. Desta forma, se faz necessário a realização de novos estudos para complemento da temática.

\section{REFERÊNCIAS}

1. Ministério da Saúde (BR), Secretaria de Vigilância em Saúde, Departamento de Vigilância das Doenças Transmissíveis. Manual de recomendações para o controle da tuberculose no Brasil. Brasília: Ministério da Saúde [Internet] 2019 [cited 2021 feb 25]. Available from: https://bvsms.saude.gov.br/bvs/publicacoes/m anual_recomendacoes_controle_tuberculose brasil_2_ed.pdf

2. Silva, MBP, Rodrigues, BS, Barreto, FM, Neto, AGS. Avaliação das condições socioeconômicas e epidemiológicas dos pacientes com tuberculose no Brasil entre 2012 a 2016. Jorn. Inter. Bioc. [Internet] 2018 [cited 2021 feb 25]; 3, (2). Available from: 
https://revistas.ufpi.br/index.php/jibi/article/vi ew/7336

3. WHO, World Health Organization. Global tuberculosis report 2020. Geneva: WHO, [Internet] 2020 [cited $2021 \mathrm{feb} 25$ ]. Available from:

https://www.who.int/publications/i/item/9789 240013131

4. WHO, World Health Organization. Global tuberculosis report 2016. Geneva: WHO [Internet] 2016 [cited 2021 feb 25]. Available from:https://apps.who.int/iris/handle/10665/2 50441

5. WHO, world health organization. Global tuberculosis report 2017. Geneva: WHO, [Internet] 2017 [cited $2021 \mathrm{feb}$ 25]. Available from:

https://www.who.int/tb/publications/global_re port/gtbr2017_main_text.pdf

6. Ministério da Saúde (BR), Boletim Epidemiológico de Tuberculose, Secretaria de Vigilância em Saúde. Brasília: Ministério da Saúde [Internet] 2020 [cited 2021 feb 25]. Available from: http://www.aids.gov.br/ptbr/pub/2020/boletim-epidemiologico-deturbeculose-2020

7. Ministério da Saúde (BR), Secretaria de Vigilância em Saúde. Sistema de Informação de Agravos de Notificação - SINAN Net. Tuberculose - casos confirmados notificados no sistema de informação de agravos de notificação - Brasília: Ministério da Saúde [Internet] 2020 [cited 2021 feb 13]. Available from:

http://tabnet.datasus.gov.br/cgi/tabcgi.exe?sin annet/cnv/tubercbr.def.

8. Ministério do Planejamento, Desenvolvimento e Gestão (BR), Instituto Brasileiro de Geografia e Estatística. Censo demográfico. Rio de Janeiro: IBGE [Internet] 2019 [cited 2021 feb 13]. Available from: https://agenciadenoticias.ibge.gov.br/agenciasala-de-imprensa/2013-agencia-denoticias/releases/25278-ibge-divulga-asestimativas-da-populacao-dos-municipiospara-2019
9. Guimarães, MHD. Tuberculose: Uma reflexão sobre o papel do enfermeiro na saúde pública. Revista Científica Multidisciplinar Núcleo do Conhecimento [Internet] 2017 [cited 2021 feb 25]; 15, 54-62. Available from:

https://www.nucleodoconhecimento.com.br/s aude/tuberculose

10. Silva, NC, Lima, EF, Costa, RMF, Araújo, EEM. Tuberculose: Assistência de enfermagem na atenção básica. Revista Electronica de Recife [Internet] 2020 [cited 2021 feb 25]; 6-1. Available from: https://reer.emnuvens.com.br/reer/article/view 1423

11. Rossoni, R, Lima, EFA, Sales, CMM et al. Protocolo de enfermagem para o paciente com tuberculose. Rev Enferm UFPE Online. 2016; 10(2):464-74. DOI: 10.5205/reuol.8557-74661-1-sm1002201612.

12. Guimarães, TMR, Amorim, CT, Barbosa EFF, et al. Cuidados de enfermagem a um paciente portador de tuberculose pulmonar e comorbidades: relato de caso. Rev Fund Care Online. 2018; 10(3):683-689. DOI: http://dx.doi.org/10.9789/ 5361.2018.v10i3.683-689.

2175-

13. COFEN. Conselho Federal de Enfermagem (COFEN). Resolução cofen358/2009: Dispõe sobre a sistematização da assistência de enfermagem e a implementação do processo de enfermagem em ambientes, públicos ou privados, em que ocorre o cuidado profissional de enfermagem, e dá outras providências. Brasília, COFEN [Internet] 2009 [cited $2021 \mathrm{feb} 25$ ]. Available from: http://www.cofen.gov.br/resoluo-cofen3582009_4384.html

14. Alves, HLC, Lima, GS, Albuquerque, GA, Gomes, EB, Cavalcante, EGR, Amaral, M. Uso das teorias de enfermagem nas teses brasileiras: estudo bibliométrico. Cogitare Enferm. 2021; 26:e71743 DOI: dx.doi.org/10.5380/ce.v26i0.71743.

15. Pires, AF, Santos, BN, Santos, PN, Brasil VR, Luna AA. A importância da Teoria do Autocuidado de Dorothea E. Orem no cuidado de enfermagem. Rev. Rede Cuid. 
Saúde. [Internet] 2015 [cited 2021 feb 25]. Available from: http://publicacoes.unigranrio.edu.br/index.php /rcs/article/view/2533

16. Silva, MM, Moreira, MC. Desafios da sistematização da assistência de enfermagem em cuidados paliativos no câncer: uma perspectiva da complexidade. Rev Eletron Enferm [internet]. 2010 [cited 2021 feb 20]; 12 (3): 483-90. Available from: https://revistas.ufg.br/fen/article/view/7274

17. Guimarães, J, Santos, L, Barreto, P, Melo, L, Andrade, J, Costacurta, FJPS. Panorama do processo de enfermagem no Brasil. REAID [Internet] 2020 [cited 2021 mar 16]; 94(32):e20080. Available from: https://revistaenfermagematual.com/index.ph $\mathrm{p} / \mathrm{revista/article/view/926}$

18. Sousa, LMM, Firmino, CF, MarquesVieira, CMA, Severino, SSP, Pestana, HCFC. Reviews of the scientific literature: Types, methods and applications in nursing. Rper. 2018; 1(1): 46-54. DOI: 10.33194/rper.2018.v1.n1.07.4391.

19. Ursi, ES, Gavão, CM. Perioperative prevention of skin injury: An integrative literature review. Ver Latino-Am Enfermagem. 2006; 14(1):124-31. DOI: 10.1590/s0104-11692006000100017.

20. Stillwell, SB, Fieout-Overholt, E, Melnyk, BM, Williamson, MKM. Evidence-based practice, step by step: Searching for the evidence. Am J Nurs. 2018; 110(5):41-7. DOI: 10.1097/01.naj.0000372071.24134.7e.

21. Critical Appraisal Skills Programme. CASP Checklist: 10 questions to help you make sense of a systematic review [Internet]. Oxford: CASP; 2018 [cited 2020 June 03]. Available from: https:// casp-uk.net/wpcontent/uploads/2018/01/CASP-SystematicReview-Checklist_2018.pdf

22. Neto, VLS, Silva, RAR, Fonseca, IA, et al. Diagnóstico de enfermería para los pacientes con tuberculosis en la unidad de enfermedades infecciosas. Cultura de los Cuidados (edición digital). 2019; 54. DOI: Http://dx.doi.org/10.14198/cuid.2019.54.29
23. Oliveira, MR, Almeida, PC, Moreira, TMM, Torres, RAM. Nursing care systematization: perceptions and knowledge of the brazilian nursing. Rev Bras Enferm [Internet] 2019 [cited 2021 mar 26]; 72(6):1547-53. Available from: https://www.scielo.br/pdf/reben/v72n6/00347167-reben-72-06-1547.pdf

24. Carvalho, CF, Ponce, MAZ, SilvaSobrinho, RA, Mendez, RDR, Santos, MA, Santos, EM et al. Tuberculosis: Knowledge among nursing undergraduate students. Rev. Bras. Enferm [Internet] 2019 [cited $2021 \mathrm{feb}$ 26]; 72( 5 ): 1279-1287. Available from:

25. Gautério-abreu, DP, Ávila JA, Santos, SSC, Ilha, S, Silva, BT. Contribuições do estudo de caso para o cuidado de enfermagem: um relato de experiência. Rev Enferm UFPE Online [Internet] 2016 [cited 2021 feb 26]; 10(3):1149-54. Available from: https://periodicos.ufpe.br/revistas/revistaenfer magem/article/view/11070

26. Andrade, SR, Andriela, BR, Talita, P, Schmit,t MD, Ferreira, A, Xavier, ACA. O estudo de caso como método de pesquisa em enfermagem: uma revisão integrativa. Texto Contexto Enferm [internet] 2017 [cited 2021 feb 27]. Available from: https://www.scielo.br/pdf/tce/v26n4/01040707-tce-26-04-e5360016.pdf

27. Alves, TEA, Vieira, AN, Guedes, MVC, Brilhante, CKCS, Oliveira, CJL, Dantas, SGM. Diretrizes de enfermagem na assistência pré-hospitalar para urgências/emergências cardiovasculares. Enferm. Foco [Internet] 2019 [cited $2021 \mathrm{feb}$ 26]; 10 (5): 173-178. Available from: https://pesquisa.bvsalud.org/portal/resource/pt /biblio-1097704

28. Tavares, DS, Souza, M, Zamberlan, C, Matumoto, S, Moreschi, C, Correa, AMG. Construção e validação de um histórico de enfermagem para consulta pré-natal. Enferm. Foco Internet] 2019 [cited 2021 feb 26]; 3542. Available from: http://revista.cofen.gov.br/index.php/enferma gem/article/view/2333

29. Felipe, TRL, Spiri, WC. Construção de um instrumento de passagem de plantão. 
Enferm. Foco [Internet] 2019 [cited $2021 \mathrm{feb}$ 26]; 76-82. Available from: http://revista.cofen.gov.br/index.php/enferma gem/article/view/2451

30. Linhares, SRS, Paz, EPA. Tratamento da tuberculose na estratégia saúde da família: olhar do profissional. Enferm. Foco [Internet] 2019 [citado 2021 feb 26]; 10 (5): 179-184. Available

from:

http://revista.cofen.gov.br/index.php/enferma gem/article/view/2407

31. Melo, LSO, Oliveira, EN, Neto, FRGX, Viana, LS, Prado, FA, Costa, JBC. Passos e descompassos no processo de cuidado aos portadores de tuberculose na atenção primária. Enferm. Foco [Internet] 2020 [cited 2021 feb 26]; 11 (1): 136-141. Available from: http://biblioteca.cofen.gov.br/cuidadoportadores-tuberculose-atencao-primaria/

32. Beserra, PJF, Gomes, GLL, Santos, MCF, Bittencourt, GKGD, Nobrega, MML. Scientific production of the international classification for nursing practice: A bibliometric study. Rev Bras Enferm [Internet] 2018 [cited 2021 mar 26]. Available from:

https://www.scielo.br/pdf/reben/v71n6/0034-

7167-reben-71-06-2860.pdf

33. Classificação Internacional para a Prática de Enfermagem (CIPE®): Versão 2019-2020 [recurso eletrônico] / Organizadora, Garcia TR; Tradução: Garcia, TR, Nóbrega $M M L$, Cubas, MR; Revisão técnica: Nóbrega MML, Cubas, MR. Porto alegre: Artmed, 2020.

34. Hirano, GSB, Lopes, CT, Barros, ALBL. Development of research on nursing diagnoses in brazilian graduate programs. Rev Bras Enferm [Internet] 2019 [cited $2021 \mathrm{mar}$ 16]; 72(4):978-85. Available from: https://www.scielo.br/pdf/reben/v72n4/00347167-reben-72-04-0926.pdf

35. Guimarães, GL, Goveia, VR, Mendoza, IYQ, Souza, KV, Guimarães, MO, Matos, SS. Contribuição da teoria de horta para crítica dos diagnósticos de enfermagem no paciente em hemodiálise. Português/inglês Rev Rnferm UFPE Online [Internet] 2016 [cited 2021 mar 16] 10(2):554-61. Available from: https://periodicos.ufpe.br/revistas/revistaenfer magem/article/viewFile/10989/12339
36. Sinha, P, Davis, J, Saag, L, Wanke, C, Salgame, P,Mesick, J et al. Subnutrição e Tuberculose: Implicações para a Saúde Pública, The Journal of Infectious Diseases. 2019; 219 (9), 1356-1363. DOI: https://doi.org/10.1093/infdis/jiy675

37. Sinha, P, Hochberg, NS. Controle da desnutrição em estudos epidemiológicos da tuberculose. The Lancet Infectious Diseases [Internet] 2020 [cited 2021 feb 23]. Available from:

https://www.thelancet.com/journals/laninf/arti cle/piis1473-3099(20)30267-x/fulltext

38. Ministério da Saúde (BR), Secretaria de Atenção à Saúde. Política Nacional de Humanização. Brasília: Ministério da Saúde [Internet] 2013 [cited 2021 feb 23]. Available from:

https://bvsms.saude.gov.br/bvs/publicacoes/p olitica_nacional_humanizacao_pnh_folheto.p df

39. Xavier, PB, Peixoto, B. Sofrimento emocional em pacientes angolanos com vários tipos de tuberculose. African Journals Online (AJOL) [Internet] 2015 [cited 2021 feb 26]; 15:(2). Available from: https://www.ajol.info//index.php/ahs/article/vi ew/117511

40. Alene, KA, Clements, ACA, McBryde, ES et al. Mental health disorders, social stressors, and health-related quality of life in patients with multidrug-resistant tuberculosis: A systematic review and meta-analysis. J Infect [Internet] 2018 [cited 2021 mar 16]; 77(5): 357-367. Available from: https://pesquisa.bvsalud.org/portal/resource/pt /mdl-30036607

41. Calsin, CNE, Schlindwein, MBH, Silva, BAB. Representações sociais das pessoas com tuberculose sobre o abandono do tratamento. Rev. Gaúcha Enferm. 2015; 36(spe): 207-214. DOI: https://doi.org/10.1590/19831447.2015.esp.56723.

42. Ayana, TM, Roba, KT \& Mabalhin, MO Prevalência de sofrimento psicológico e fatores associados entre pacientes adultos com tuberculose atendidos em instituições de saúde pública nas cidades de Dire Dawa e Harar, Leste da Etiópia. BMC Public Health, 
2019;

19 ,

1392.

DOI:

https://doi.org/10.1186/s12889-019-7684-2.

43. Rosa MLJMAF. Implicações psicossociais e familiares da doença na pessoa com tuberculose. Lisboa: Universidade Aberta [Internet] 2007 [cited 2021 feb 26]. Available from: https://core.ac.uk/download/pdf/303039686.p df

44. North American Nursing Diagnosis Association. Diagnósticos de enfermagem da NANDA-I: definições e classificação 20182020. 11. ed. Porto Alegre: Artmed, 2018.

\section{Autor correspondente}

Maiana Evillyn da Silva Santos, rua Japaratuba, $n^{\circ}$ 866, bairro Santo Antônio, Aracaju-SE, CEP 49060300, telefone- (79) 988650021 e e-mail maiana_evellyn@hotmail.com

Submissão: 2021-05-12

Aprovado: 2021-07-11 